\title{
Cow's Milk Yogurt Modulate Expression of STAT5, BMI-1 and LGR-5 in Ileum of Indomethacine-Induced Inflammatory Bowel Disease (IBD) in Rats.
}

\author{
Masdiana Chendrakasih Padaga ${ }^{1, *}$, Wibi Riawan ${ }^{2}$, Nur Hamni ${ }^{1}$ \\ ${ }^{1}$ Faculty of Veterinary Medicine, Brawijaya University, \\ Malang, 65145, Indonesia. \\ ${ }^{2}$ Department of Biochemistry, Faculty of Medical Science, Brawijaya University, \\ Malang, 65145, Indonesia. \\ * Corresponding author: mpadaga@gmail.com
}

\begin{abstract}
Inflammatory bowel disease (IBD) is characterized by a chronic recurrent inflammation of the gastrointestinal tract. The environmental factors affecting IBD include diet, the use of antibiotics and nonsteroidal anti-inflammatory drugs (NSAIDs) and stress. All of which can directly or indirectly affect the epithelial barrier of intestinal that consists of the intestinal epithelial cells (IEC). The Janus kinase (JAK)-signal transducer and activator of transcription (STAT) 3 pathway is critically involved in IEC proliferation during homeostasis as well as enhance proliferation that needed for tissue repair in active IBD. Mucosal damage triggers Upd3 cytokin to activate JAK/STAT Pathways in renewing epithelium. Activation of the stem cells characterized by expression of LGR5+ and BMI-1. Effect of cow's milk yogurt on the expression of STAT5, BMI-1 and LGR5+ in ileum of Indomethacine-Induced IBD rats (Rattus norvegicus) were studied. The IBD rats were then orally administrated with cow's milk yogurt in daily dose of 300, 600 and $900 \mathrm{mg} / \mathrm{kg}$ body weight for 14 days. The effect of yogurt treatment on Stat5, BMI-1 and LGR-5 of rats ileum were evaluated using immunohistochemistry method. The results showed that the expression of Stat5, BMI-1 and LGR-5 increased significantly after yogurt administration. Structurally also detected there were regeneration of the epithelial cells and repairing the epithelial damage. The current study suggests that cow's milk yoghurt treatment was able to regulate stem cell proliferation and repair intestinal damage in Indomethacine-Induced IBD rats model.
\end{abstract}

Key words : Inflammatory bowel disease (IBD), JAK/STAT, Bmi-1, Lgr5,yogurt

\section{INTRODUCTION}

Induction of indomethacin to animal model rats (Rattus norvegicus) results in mucosal ileal damage. This damage will trigger an UPD3 cytokine to activate the JAK / STAT pathway to initiate the epithelial regeneration process. The regeneration process begins with stem cell proliferation to replace damaged epithelial cells. Signals on the JAK / STAT pathway are important pathways in the proliferation process, especially in response to mucosal damage. Activation of JAK / STAT is assessed from Stat5 expression. Stem cell activation is characterized by the expression of LGR5 + and BMI1. LGR5 + as a stem cell indicator that continues proliferating in the state of homeostasis, while BMI1 as a stem cell marker that is in a quiscent state and is activated in damage state and can serve as a replacement for damaged LGR5 + cells (Villarino et al., 2015). The intestinal stem cells are located at the base of the crypt, these stem cells adjacent to the Paneth cells and surrounded 
by a stromal microenviroment. The stroma consists of several cell components, i.e extracellular matrix, fibroblasts, myofibroblasts, smooth muscle cells, neural cells, endothelial cells, lymphocytes and macrophages.

Yogurt contains probiotic bacteria which can protect the gastrointestinal mucosa and as immunomodulator that can prevent inflammatory in the gastrointestinal tract. Yogurt also as source of bioactive peptides that provide biological activity as an immunomodulator that plays a role in the intestinal barrier function (IBF) and contain antioxidant compounds such as selenium, glutathione peroxidase, and thioredoxin reductase that can protect the tissue from stress oxidative damage. Extracellular proteins produced by probiotics have an important role in the molecular communication between probiotics and intestinal epithelial cells. Protein secreted by probiotics in the gastrointestinal tract include IL 10 and protein p40. IL-10 will activate the JAK / STAT path and p40 will activate the EGFR path. Activation of these two pathways by probiotics is expected to accelerate the regeneration process. Treatment of IBD with probiotics is expected to affect the niche of intestinal stem cells and activate intestinal stem cells, hence, proliferation and differentiation can occur more quickly.

\section{MATERIALS AND METHODS}

\section{Yogurt preparation}

Yogurt was made from cow's milk fermented with starter (Yogourtmet )containing three Lactic acid bacteria (Streptococcus thermophilus, Lactobacillus acidophilus, dan Lactobacillus bulgaricus). The yogurt was freeze-dried and kept at $-20^{\circ} \mathrm{C}$ until used.

\section{Animal Model}

All procedures were carried out in accordance with conventional guidelines for experimentation with animals. Twelve-week old male rats (Rattus norvegicus) strains Wistar were used. The rats were divided into 5 groups, i.e negative control (A), positive controlIBD, the rats were induced with indometacin $15 \mathrm{mg} / \mathrm{Kg} \mathrm{BW}(\mathrm{B})$, IBD rats treated with yogurt $300 \mathrm{mg} / \mathrm{kg}(\mathrm{C})$, IBD rats treated with yogurt $600 \mathrm{mg} / \mathrm{kg}$ (D), IBD rats treated with yogurt $900 \mathrm{mg} / \mathrm{kg}(\mathrm{E})$, and were housed in groups of four per cage, as a number for replication, in a regulated environment with a $12 \mathrm{~h}$ light/dark cycle. The treated IBD rats received, by oral administration, using a canula, a daily dose of yogurt in daily dose of 300 , 600 and $900 \mathrm{mg} / \mathrm{kg}$ body weight dissolved in reverse osmosis water for 14 days.

\section{Histopathology of ileum}

The ileum tissues stained with haematoxylin and eosin (H\&E) dye and examined under a high power microscope.

\section{STAT5, BMI-1, LGR-5 expression}

Expression of STAT5, BMI-1, LGR-5 in ileum were analyzed by immunohistochemistry method using primary antibody anti-rat STAT5, BMI-1 and LGR-5 followed by anti rabbit labelled biotin secondary antibody and react with SA-HRP. Positive reactions were visualized by addition of DAB Chromagen substrate (3,3-diaminobenzidine tetrahydrochloride) and counterstain with mayer hematoxylen. 


\section{RESULTS AND DISCUSSION}

\section{Histopatology of ileum}

Cow's milk yogurt can improve the histopathologic of rat ureum IBD indomethacin induction model shown in Figure 1.

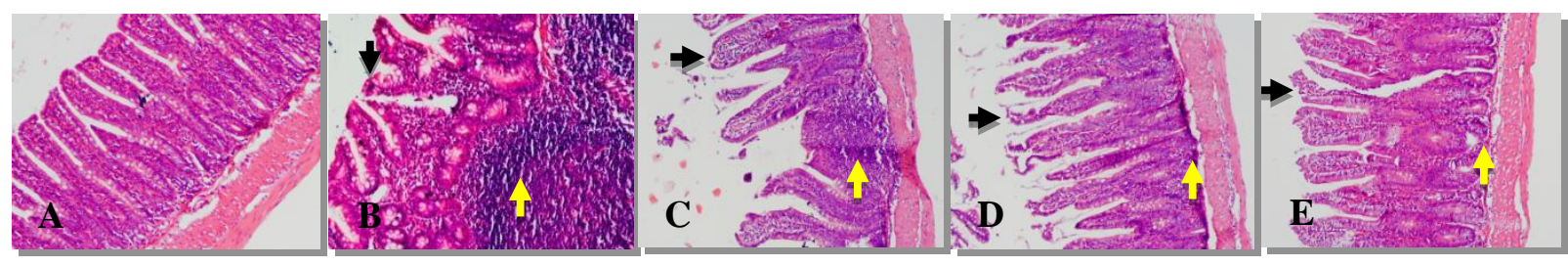

Figure 1. Representative images of H\&E-stained ileum sections illustrate specifics of inflammatory cell infiltrates $(\uparrow)$ and epithelial changes $(\uparrow)$. (A) normal ileum showing no alterations in the overall intestinal tissue architecture. (B) ileum of IBD rats showing mucosal infiltration of mixed inflammatory cells and changes in epithelial structures. (C) ileum of IBD rats treated with yogurt $300 \mathrm{mg} / \mathrm{kg} \mathrm{BW}$. (D) ileum of IBD rats treated with yogurt $600 \mathrm{mg} / \mathrm{kg} \mathrm{BW}$. (E) ileum of IBD rats treated with yogurt $900 \mathrm{mg} / \mathrm{kg} \mathrm{BW}$

Normal ileal histology comprises a densely packed mucosa, columnar epithelial cells with goblet cells, submucosal layer, mucosal muscular layer, muscular layer and serous layer (Erben et al., 2014). Induction of indomethacin $15 \mathrm{mg} / \mathrm{kg}$ BW for 24 hours resulting in ileal inflamation characterized microscopically by diffuse inflammatory lesions with regular shortening of the villi, discontinuity of epithelial cells and infiltration of inflammatory cells in submucosa layer. Treatment of IBD model rats with cow milk yogurt at a dose of 300,600 and $900 \mathrm{mg} / \mathrm{kg} \mathrm{BW}$ in IBD model rats showed marked improvement of intestine structure and decreased infiltration of inflammatory cells in submucosa. Cow milk yogurt has the potential to improve ileal damage in IBD model rats. The potential of cow's milk yogurt as a candidate for IBD therapy is due to the presence of lactic acid bacteria in yogurt. The yogurt contains three lactic acid bacteria namely Lactobacillus acidophilus, Streptococcus thermophilus, and Lactobacillus bulgaricus. These beneficial bacteria in the gastrointestinal tract can be an immunomodulator that stimulate intestinal mucosal immunity in order to strengthen intestinal mucosal barrier, increase the production of antiinflammatory cytokines and reduce the production of proinflammatory cytokines as well as reduce the inflammatory response.

\section{STAT5, BMI-1 DAN LGR-5 expression in Ileum of IBD model rats}

Intestinal epithelium is a network that undergoes regular and constant changes. This routine change should still ensure the absorption of nutrients for the body needs (Hsu dan Fuchs, 2012). Intestinal epithelium is composed of villi and crypt. The crypt of ileum contain stem cells that proliferate, undifferentiate, and rapidly cycling. There are 2 types of intestinal stem cell, ie cell populations expressing BMI1 and LGR5. The cell expressing BMI1 is in the +4 position, whereas LGR5 is at the base of the crypt between the Paneth cells. BMI1 cell is a quiescence stem cell population and is not an active stem cell. Cells with positive LGR5 expression are active stem cells. 
Indomethacin induction of $15 \mathrm{mg} / \mathrm{kg} \mathrm{BW}$ in Wistar rats result the ileal mucosa damage. This stimulate the body's response to regenerate (Fig. 2B). Treatment with yogurt in the dose of $300 \mathrm{mg} / \mathrm{kg}$ bw (Fig. 2C), $600 \mathrm{mg} / \mathrm{kg}$ bw (Fig.2D) and $900 \mathrm{mg} / \mathrm{kg} \mathrm{bw} \mathrm{(Fig.2C)}$ showed mucosal repairing. This mucosal repair process begins with stem cell proliferation, followed by differentiation. The damaged epithelial cells will be replaced with new mature cells. The body response is achieved by complex interregulation between the immune response, intestinal microbiota and stem cell activity. Mucosal damage causes the expression of UPD3 cytokines. This cytokine activates the JAK / STAT pathway, resulting in the proliferation and differentiation of intestinal stem cells (Yan et al., 2014).

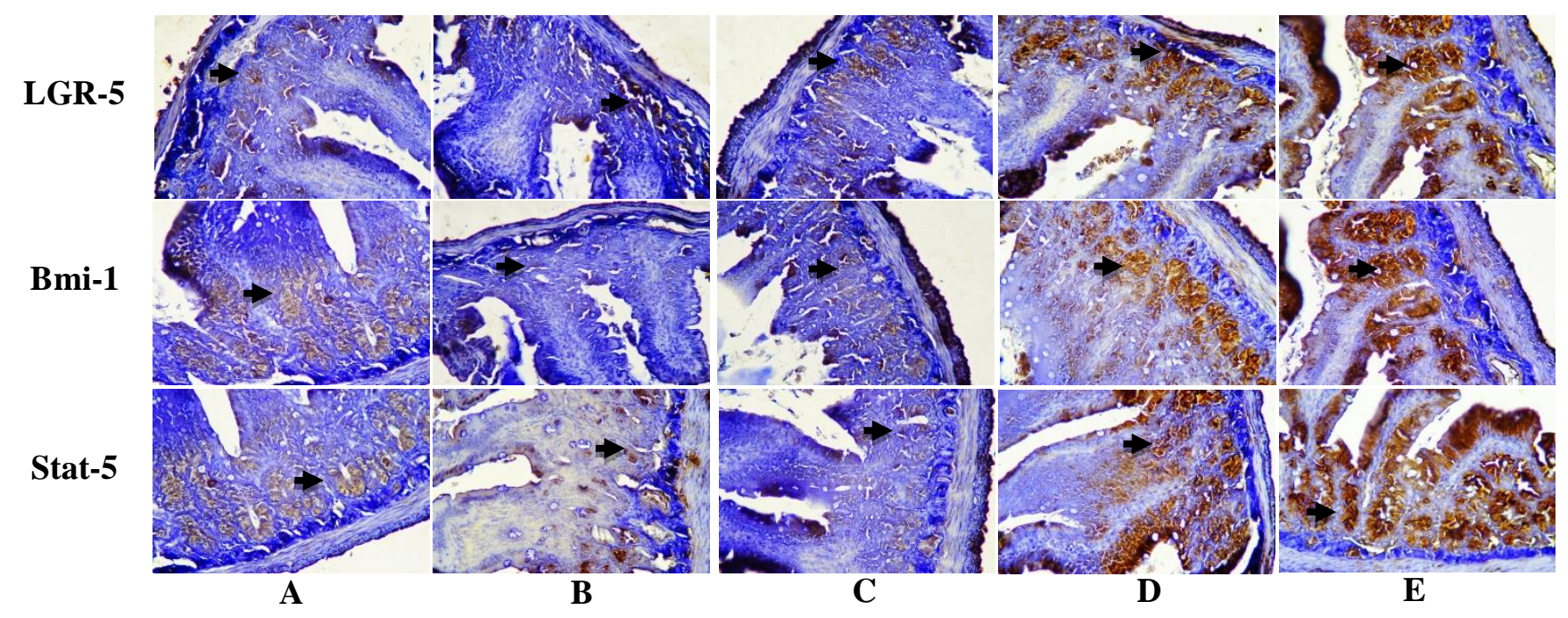

Figure 2. Representative immunohistochemically stained ileal sections using anti-rat STAT5, BMI-1 dan LGR-5, illustrate crypt epithelial (A) normal ileum.(B) ileum of IBD rats (C) ileum of IBD rats treated with yogurt $300 \mathrm{mg} / \mathrm{kg} \mathrm{BW}$. (D) ileum of IBD rats treated with yogurt $600 \mathrm{mg} / \mathrm{kg}$ BW. (E) ileum of IBD rats treated with yogurt $900 \mathrm{mg} / \mathrm{kg} \mathrm{BW}$ 


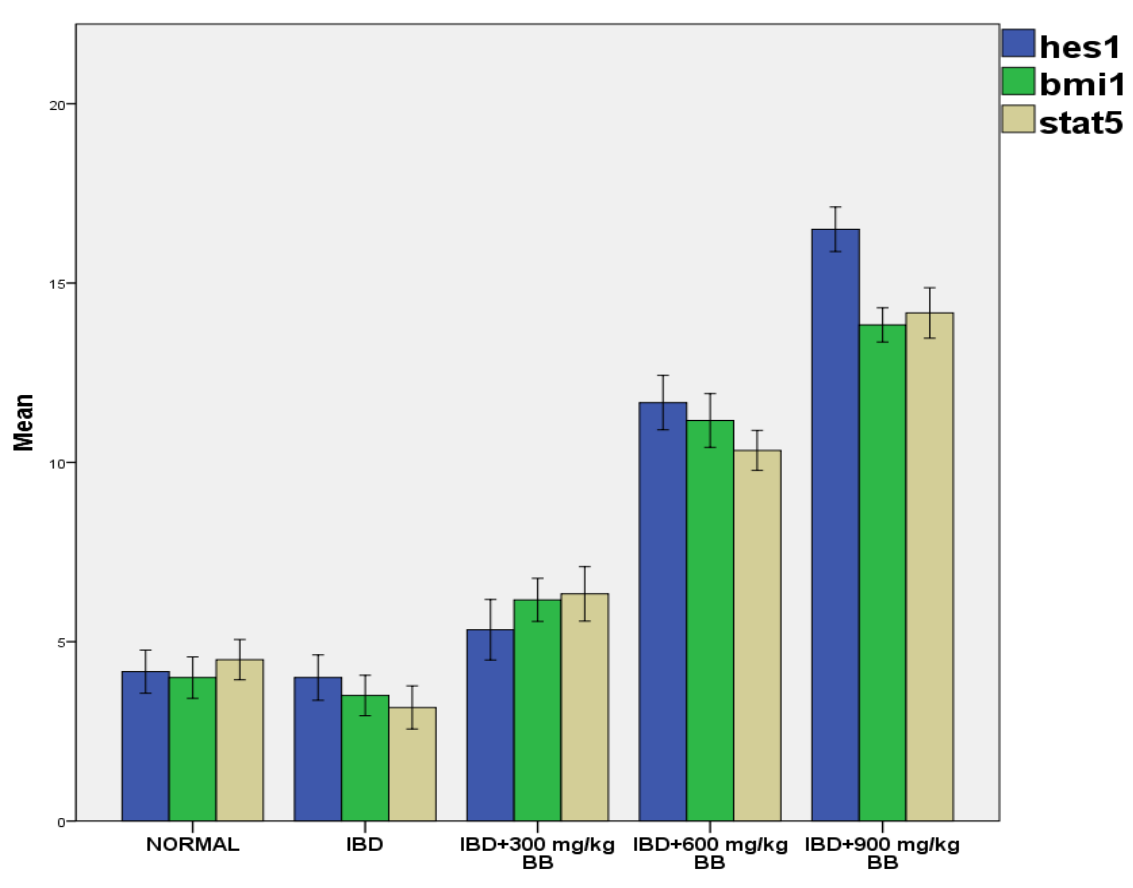

Figure 3. Representative immunohistochemically percentage area using anti-rat STAT5, BMI-1 dan LGR-5, illustrate crypt epithelial (A) normal ileum.(B) ileum of IBD rats (C) ileum of IBD rats treated with yogurt $300 \mathrm{mg} / \mathrm{kg} \mathrm{BW}$. (D) ileum of IBD rats treated with yogurt $600 \mathrm{mg} / \mathrm{kg} \mathrm{BW}$. (E) ileum of IBD rats treated with yogurt $900 \mathrm{mg} / \mathrm{kg} \mathrm{BW}$

The JAK / STAT signal path is the pathway responsible for stem cell proliferation regulation. The JAK / STAT along with JNK pathway activated stem cell proliferation and epithelial repair due to infection (Heneghan et al., 2013). The Upd3 cytokines produced from epithelial damage due to infection can activate the JAK / STAT signal path. The intestinal microbiota produces a smaller number of Upd3 cytokines, so the JAK / STAT pathway remains activated to maintain intestinal epithelial bowel homeostasis (Fuch \& Chen, 2013). Lactobacillus rhamnosus GG (LGG) also has p40 and p75 proteins. Both of these proteins regulate intestinal homeostasis and repair epithelial intestinal damage through activation of EGFR (Yan et al., 2013).

\section{REFERENCES}

Fuchs, E and Chen, T. 2013. A matter of life and death: self-renewal in stem cells. EMBO reports $14,39-48$

Heneghan, A.F, Pierre, J.F, Kudsk, K.A. 2013. JAK-STAT and intestinal mucosal immunology. http://dx.doi.org/10.4161/jkst.25530

Johansson, M.E., Ambort, D., Pelaseyed, T., Schütte, A., Gustafsson, J.K., Ermund, A. 2011. Composition and functional role of the mucus layers in the intestine.

Cell Mol Life Sci. 68:3635-41; http://dx.doi.org/10.1007/s00018-011-0822-3

Rawlings, J.S., Rosler, K.M., Harrison, D.A. 2004.The JAK/STAT signaling pathway. J. Cell Sci; 117:1281-3. http://dx.doi.org/10.1242/jcs.00963 
Boland, B.S., Sandborn, W.J., and Chang, J.T. 2014. Update on Janus Kinase Antagonists in Inflammatory Bowel Disease. Gastroenterol Clin North Am. 43(3): 603-617. doi:10.1016/j.gtc.2014.05.011

Yan,F., Liu,L., Dempsey,P.J., Tsai,Y.H., Raines, E.W., Wilson, C.L., Cao,H., Cao, Z., Liu,L.S. and Polk,D.B. 2013. A Lactobacillus rhamnosus GG-derived Soluble Protein, p40, Stimulates Ligand Release from Intestinal Epithelial Cells to Transactivate Epidermal Growth Factor Receptor. 2013. The Journal of Biological Chemistry 288 (42) : 30742-30751

Daren,L., Nguyen, D.D and Mizoguchi, E. 2013.Animal models of ulcerative colitis and their application in drug research. Drug Design, Development and Therapy 7 (1): 1341-1357. doi:10.2147/DDDT.S40107.

Nielsen,O.H. 2014. New strategies for treatment of inflammatory bowel disease. doi: 10.3389/fmed.2014.00003

Erben1,U., Loddenkemper, C., Doerfel,K., Spieckermann1,S., Haller,D., Heimesaat,M.M., Zeitz,M., Siegmund,B., Kühl, A.A. 2014. A guide to histomorphological evaluation of intestinal inflammation in mouse models. Int J Clin Exp Pathol 2014;7(8):4557-4576

Rawlings, J.S., Rosler, K.M., and Harrison, D.A. 2004. The JAK/STAT signaling pathway. Journal of Cell Science 117, 1281-1283. doi:10.1242/jcs.00963

Villarino, A.V., Kanno,Y., Ferdinand, Y.R and O'Shea,J.J. 2015. Mechanisms of Jak/STAT Signaling in Immunity and Disease. J Immunol 194:21-27; doi: 10.4049/jimmunol.1401867 